\title{
RESILIENCIA: ADOLESCENTE ANTE ABUSO SEXUAL Y NEGLIGENCIA FAMILIAR
}

\section{RESILIENCE: TEENAGER FRONT SEXUAL ABUSE AND FAMILY NEGLECT}

Brissia Stephanie Fuentes Carmen*, Santos Noé Herrera-Mijangos*, Andrómeda Ivette Valencia Ortiz*, Ana Olivia Ruíz Martínez**

Universidad Autónoma del Estado de Hidalgo*, Universidad Autónoma del Estado de México**; México

Correspondencia: briss0808@gmail.com

\section{RESUMEN}

Las consecuencias que generan la complicidad y negligencia ante el abuso sexual pueden ser experiencias sumamente traumáticas para cualquier niño o adolescente, no obstante, a pesar de su exposición a situaciones de riesgo, hay personas que no parecen desarrollar problemas. El principal objetivo de esta investigación describe la dinámica familiar que condujo al abuso sexual; así como el comportamiento resiliente de una adolescente. Se presenta una historia de vida, de una mujer que fue víctima de abuso sexual, y logró reponerse ante la falta de apoyo de su familia. El diseño es de caso único, lo que aquí se interpreta no puede ser generalizado. La metodología de esta investigación es de corte cualitativo, se utilizó el método inductivo-analítico, de modo que nos permite observar los detalles de las vicisitudes y resistencias de hechos desafortunados. Los resultados nos permitieron adentrarnos en la dinámica familiar y mostraron cómo los padres descuidaron y pusieron en riesgo al adolescente. Finalmente, la protagonista mostró sus fortalezas y virtudes desarrolladas ante las adversidades de su vida. 
Palabras clave: abuso sexual, dinámica familiar, adolescencia, negligencia parental y resiliencia.

\section{ABSTRACT}

Sexual abuse can be an extremely traumatic experience for anyone, however, dispite complicity and neglect of some family members, there are people who do not seem to develop problems. The main objective of this research, describes the family dynamics that led sexual abuse; as well as the resilient behavior of a teenager. A life story is presented in this word, it is about a woman who was a victim of sexual abuse. She managed and recovered from the lack of support from her family. It is a single-case, and it cannot be generalized. It is a qualitative methodology research, the inductive-analytical method was used, because it allows us to observe the details of the vicissitudes and resistances of unfortunate events. The results allowed us to get into family dynamics and showed neglected parents that put in danger to their adolescent daughter. Finally, the protagonist showed her strengths and virtues developed in her life.

Key words: sexual abuse, family dynamics, adolescence, parental neglect and resilience.

\section{INTRODUCCIÓN}

El abuso sexual de niños y adolescentes no es un problema reciente. A lo largo de la historia se ha manifestado y reconocido a nivel nacional e internacional por los efectos a largo plazo de las víctimas (Timmerman \& Schreuder, 2014). La Organización Mundial de la Salud (OMS, 2020) calculó que hasta mil millones de niños y adolescentes de entre 2 y 17 años fueron víctimas de abusos físicos, sexuales, emocionales o de abandono. En este sentido, la prevalencia de abusos y violencia es y ha sido a través de los tiempos una actitud presente durante toda la vida afectando a infantes 
y adolescentes hasta la edad adulta. La mayoría de los casos de violencia sexual en México son contra las mujeres, las jóvenes, las niñas y los niños (Fondo de las Naciones Unidas para la Infancia [UNICEF], 2019).

En la República Mexicana, alrededor de 711,226 mujeres fueron víctimas de delitos sexuales 40,303 sufrieron una violación sexual y 682,342 mujeres fueron víctimas de hostigamiento, manoseo, exhibicionismo o intento de violación (Instituto Nacional de Estadística y Geografía [INEGI], 2019). De acuerdo con Pinheiro y Fortunato (2019) la violencia sexual hace referencia a cualquier acto o conducta dirigida a la satisfacción sexual de un adulto o adolescente con una diferencia de edad significativa para la víctima, y puede o no incluir contacto físico y penetración.

En la mayoria de los casos el abuso sexual es perpetuado por integrantes de la misma familia. De acuerdo con Pinheiro y Fortunato (2019) el abuso sexual hace referencia a cualquier acto o conducta dirigida a la satisfacción sexual de un adulto o adolescente con una diferencia de edad significativa para la víctima, y puede o no incluir contacto físico y penetración. En este contexto, es importante destacar a la familia como un grupo social, dentro del cual cada miembro tiene la oportunidad de desarrollarse. La familia reitera el valor que los miembros de los sistemas familiares tienen sobre los niños y adolescentes ante el abuso (Losada, 2015). Así, este valor es explícito no sólo en la participación, orientación y supervisión que los adultos responsables dirigen a los niños y adolescentes, sino también porque, dependiendo de la estructura y la dinámica familiar, este núcleo puede ser un factor de riesgo para la ocurrencia de rechazo y violencia (Pinheiro \& Fortunato, 2019).

De acuerdo con Sánchez, Aguirre, Solano y Viveros (2015) definen la dinámica familiar como el clima relacional que se establece entre los 
integrantes de la familia. Es decir, como el resultado de la vida cotidiana que establece la familia, la interacción entre sus miembros y los procesos internos que conforman un ambiente propio y genuino (Agudelo, 2010). De acuerdo con Gallego, Pino, Álvarez, Vargas y Correa (2019), en la dinámica familiar, tanto los sujetos como las familias son estudiados en función de sus pautas de organización, es decir, sus aspectos constitutivos: los roles, la autoridad, las normas, los límites, las relaciones afectivas la comunicación y el uso del tiempo libre. La importancia de conocer todas estas características radica en el fenómeno que representa la familia como fuente de salud o enfermedad, es decir, como un apoyo u amenaza.

En cuanto a la amenaza, Franco y Ramírez (2016) manifiestan que la negligencia familiar es una de las formas de violencia menos evidenciadas y expresadas, el cual es un tema que no ha sido adecuadamente analizado en cuanto a su frecuencia y repercusiones. De este modo, los padres negligentes son aquellos que expresan un bajo nivel de afecto hacia sus hijos, se comunican poco con ellos y las prácticas de crianza son deficientes. De hecho, no se involucran con lo que les suceda en ningún ámbito, son en general distantes y fríos (García, Rivera, \& Reyes, 2014).

Para Quiceno, Mateus, Cardenas, Villareal y Vinaccia (2013) las consecuencias que generan la complicidad y negligencia de los progenitores ante el abuso pueden ser experiencias sumamente traumáticas, no obstante, a pesar de su exposición a situaciones de abuso, hay personas que tienen como factor de protección la resiliencia y parecen no desarrollar problemas. Si bien es sabido, el abuso sexual es una amenaza real para cualquier niño o adolescente, también es cierto que no toda experiencia produce daños psicológicos y sociales en las víctimas (Domhardt, Münzer, Fegert., \& Goldbeck, 2015). La importancia de estudiar el abuso y malos tratos en la 
adolescencia radica en que, a pesar de su exposición a situaciones de riesgo, algunos sujetos no parecen desarrollar problemas que podrían derivarse de estas experiencias, consiguiendo una vida sana, plena e integrada (Villegas \& Mallor, 2017).

Para aprender cómo algunas personas viven vidas plenas a pesar de haber vivido experiencias traumáticas, es necesario entender la resiliencia y la postura de la psicología positiva. González-Arratia (2016), menciona a la resiliencia como un proceso dinámico donde el individuo traumatizado se sobrepone y reconstruye. Es decir, no representa la inmunidad o impermeabilidad al trauma sino la capacidad de recuperarse en las experiencias adversas. Sin embargo, para Piña (2015) no es una mera resistencia a la adversidad sino que hace referencia a la capacidad de crecer o desarrollarse en los contextos difíciles.

Desde modelos más optimistas, se considera que la persona es fuerte y activa, con una capacidad natural de resistir y rehacerse a pesar de las adversidades. Esta concepción se enmarca dentro del marco de la psicología positiva, y pretende comprender los procesos y mecanismos que subyacen a las fortalezas y virtudes del ser humano (Astorga, Castro, Cabaco, \& Velasco, 2014). La psicología positiva mantiene que el ser humano tiene capacidad para adaptarse y recuperarse de situaciones adversas. Es decir, vivir una experiencia traumática y volver a construir su forma de entender el mundo y su sistema de valores (Cala, 2020).

Es importante reconocer que los avances en la investigación de la resiliencia, han puesto de manifiesto que las emociones positivas son mecanismos habituales para enfrentar situaciones adversas. Bajo esta perspectiva, el desarrollo del estudio de la resiliencia se ha convertido en 
una necesidad que se puede desarrollar a cualquier edad y variar de una persona a otra. El presente artículo demuestra de forma contundente que la resiliencia es una respuesta común y su aparición no indica patología, sino un ajuste saludable a la adversidad. Con la finalidad de aportar nuevos conocimientos para ayudar, a las personas o grupos a resolver problemas de salud y mejorar la calidad de vida (Noriega, Arjona., \& Noriega, 2015). Considerando lo presentado en los párrafos previos, el objetivo central de este artículo es describir la dinámica familiar que propició el abuso sexual; así como la conducta resiliente de una adolescente. Se busca responder las siguientes preguntas: ¿cómo es la dinámica entre los miembros de la familia?, ¿cuáles son los riesgos sociales a los que se enfrenta una adolescente marginada? y ¿qué tipo de factores resilientes emplea la adolescente?

\section{MÉTODO}

\section{Tipo de investigación}

Esta investigación es de corte cualitativo, se utilizó el método inductivoanalítico, de modo que, la metodología ofrece información sobre las vivencias, percepciones, sentimientos y emociones de las personas (Balcázar, González-Arratia, Gurrola, \& Moysén, 2013). Se presenta una historia de vida que, para los propósitos de la investigación, se redactó como una novela que permite involucrarse en el mundo interior vivido y experimentado (Villegas \& González, 2011). La realización de la novela requiere de la descomposición de la realidad para convertirla en objeto de estudio; la indagación se hace desde un enfoque situacional, aquí y ahora, y desde un enfoque histórico, cómo fueron y llegaron a ser lo que son (Remedi, 2004). El diseño es de caso único, lo que aquí se interpreta no puede ser generalizado. 


\section{Participante}

Mujer víctima de abuso sexual durante la niñez y adolescencia. La mujer tiene 20 años de edad, una carrera universitaria y su nivel socioeconómico es medio bajo. Es originaria de Pachuca de Soto donde vive actualmente.

\section{Escenario}

La entrevista se realizó en la oficina de la participante ubicada en Pachuca de Soto en marzo de 2020, a la hora y lugar que ella decidió.

\section{Consideraciones éticas}

La participante decidió colaborar voluntariamente, los fragmentos de entrevista presentados tienen un seudónimo "Regina" a fin de conservar la privacidad. Cabe destacar que se tiene grabada la autorización de la entrevistada para publicar los resultados con fines académicos.

\section{Materiales y procedimiento}

Se creó una guía de entrevista semiestructurada y se realizó una entrevista a profundidad la cual fue audio-grabada. Una vez obtenida la entrevista, se transcribió y redactó la novela con la información proporcionada en la entrevista. Cabe destacar que durante la redacción se sostuvieron pláticas con "Regina" para aclarar dudas sobre la información que proporcionó durante la entrevista. Cuando la novela se concluyó, se presentó a Regina para agregar, quitar y modificar información que considerara pertinente. Es prudente aclarar que los investigadores sólo se convierten en relatores que construyen la novela que representa una realidad narrada de la participante y es similar algunas historias de mujeres jóvenes en varias partes del mundo. 


\section{Análisis de datos}

Se analizó la entrevista a profundidad y artículos teóricos. La entrevista se transcribió e identificaron ejes y categorías tanto preestablecidas como las emergentes a partir de la narración de la participante (Cuevas, 2016). Las preestablecidas se refieren a la dinámica familiar, los riesgos sociales y los factores resilientes. Las emergentes fueron aquellos temas relacionados con las costumbres, la rivalidad entre hermanas, la vulnerabilidad y riesgo por el rol que ocupa en la familia así como la resistencia al daño, entre otros. Una vez obtenidos los ejes y categorías, se alinearon las respuestas con los objetivos de la investigación. Este tipo de análisis es "trabajo artesanal" porque no se utiliza software que generaliza resultados, ya que se consideró que su uso generaría que la investigación se tornara con un enfoque rígido. La selección de las partes de las entrevistas depende de una búsqueda en la narración de la participante y sirvió para crear la novela en la que el investigador dio voz a la participante. Cabe señalar que, aunque los resultados se presentan de una manera literaria, el contenido de la novela respetó en todo momento lo expresado por la participante.

\section{RESULTADOS}

\section{Novela: la presencia de la ausencia}

Regina nació un año antes de comenzar el presente siglo en Pachuca de Soto, Hidalgo, México. Su familia, de nivel socioeconómico medio bajo, está compuesta por cuatro integrantes: padres, hermana y ella, la hija menor. Como en la mayoría de las familias, comúnmente los nombres de los hijos son seleccionados con base en eventos, experiencias, sueños e ideales de los padres. Su hermana mayor, Alejandra heredó el nombre del abuelo paterno por coincidencias de la vida, Regina comenta: "Mi hermana tiene el nombre de mi abuelo paterno porque cuando nació, mi abuelo 
murió. Mis padres decidieron ponerle Alejandra en honor al abuelo". El nombre de las hermanas marcó desde un inicio las diferencias en el trato que recibieron por parte de los padres. Regina afirma que a ella le pusieron "el nombre que quedó, un nombre que habían pensado originalmente para la hermana".

A simple vista, las hermanas recibieron el mismo trato por parte de la madre. Su progenitora estuvo presente, en el aspecto moral, aunque Regina después de la secundaria no obtuvo apoyo económico para continuar con sus estudios. La relación y vínculo con el padre, no fue agradable, ha sido "un tanto hostil y extraño", distante, de poco afecto hacia ella comparado con su hermana.

Regina recuerda que su hermana Alejandra "salía cada año en el cuadro de honor y obtenía obsequios" por sus logros académicos. Regina intentó seguir el ejemplo y en tercer año de secundaria logró su cometido, obtuvo el primer lugar por lo que esperaba el reconocimiento del padre. Recuerda que en su escuela se "entregaban reconocimientos a los alumnos con mejor promedio". Regina estaba muy emocionada e invitó a su papá para que la acompañara en tan importante evento "pero mi papá nunca llegó, no quiso acompañarme". La relación familiar con el tiempo se desgastó por las peleas constantes con su hermana, la frialdad del padre y la falta de empatía de los progenitores, cada evento contribuyó para fracturar la relación familiar.

Los padres de Regina han sido como tantos otros que han querido imponer a sus hijos sus deseos e ideales no cumplidos. La madre sin consultarla realizó los trámites pertinentes para que asistirá a la escuela preparatoria que ellos decidieron. Tal imposición resultó en el abandono de la escuela. 
Regina reconoce que desertó dos veces como "un capricho, porque no quería esa carrera". La falta de compromiso con la escuela por parte de Regina ocasionó que sus padres dejaran de apoyarla económicamente y a partir de dicha situación Regina empezó a trabajar en la maquiladora donde laboraba su madre.

Regina sabe que dejar la escuela fue su decisión, sin embargo, cuestionó: "si mi hermana también dejó de estudiar, ¿por qué no le retiran el apoyo económico?" Ante su interrogante, sus padres justificaban las diferencias diciendo: "tu hermana mayor necesita que la empujen para que pueda volar por sí sola". Como era de esperarse, las diferencias que hicieron los padres, provocaron discusiones y competencia entre las hermanas: "mi hermana es envidiosa con lo que me pasa, dice que le caigo mal".

A pesar de la actitud de sus padres, ella rectificó su situación académica. A los 16 años aprobó el examen único del CENEVAL y a los 17 "estudié la carrera de diseño gráfico". Regina permaneció dos años trabajando y estudiando. Cuando tenía posibilidad no dudaba en invertir tiempo con su familia a pesar de las diferencias "cuando me quedaba un poquito de dinero invitaba a mi mamá y hermana a comer o les compraba un helado" aunque sentía un desamparo. La situación desfavorable que vivió Regina en su familia, fue observada por otros miembros externos de la familia y la ubicó en una condición de vulnerabilidad y riesgo.

\section{Secreto a voces: el abuso sexual}

La mayoría de los padres hacen diferencias significativas entre sus hijos. Comúnmente están aquellos que son los consentidos, herederos y los no queridos; claro la mayoría tiende a negar que existan preferencias por parte de ellos. Este tipo de discriminación familiar interna, en ocasiones 
es evidente por la familia extensa (abuelos, tíos y primos entre otros) quienes también pueden discriminar. Es decir, si los padres tratan mal a un hijo, darán un mensaje de que cualquiera puede maltratar al niño, por el contrario, si los padres protegen al niño, están atentos de cualquier anomalía, los externos sabrán que, de haber un abuso, las consecuencias serán graves (Schützenberger, 2006).

Nuestra protagonista, aunque era la menor, fue la hija no querida de los padres. Aunque nunca se manifestó abiertamente, las acciones cotidianas dejaron ver que la hija predilecta fue la que heredó el nombre del abuelo, Alejandra, la mayor. Esta dinámica familiar fue percatada por la familia extensa, particularmente por una tía y su hijo. Regina recuerda: "cuando tenía doce años, mi primo me tocó los pechos jugando". Ante la incomodidad y asombro por el abuso, Regina compartió lo ocurrido con su madre quien tomó partido por su hija y acudió con la madre del primo para tratar el asunto, pero fue condenada por su tía, le dijo: "para que te llevas, si después, no te vas aguantar”. Como en muchas típicas familias, el asunto se ocultó y condenó a la mujer. Regina comparte que la relación con su primo había sido buena, hasta aquel momento, aunque las experiencias de abuso por parte de sus primos continuaron.

Regina recuerda borrosamente que cuando tenía 19 años, salió "de fiesta con otro de mis primos, que le pidió permiso a mi mamá". Su madre concedió la salida porque el joven seis años mayor que su hija pertenecía a la familia. Regina comenta: "salimos a tomar whisky y después vodka". Todo parecía normal esa noche, la bebida, la música, el baile y la compañía hasta que su primo le dijo: "vamos a tomarnos una cruzada". Al calor de la fiesta, Regina aceptó tomar la bebida, pero una vez que la ingirió, rompió el vaso y perdió el conocimiento: "a partir de ese momento no recuerdo 
nada, fue como si hubiera caído en un profundo sueño". Regina, perdió el control de sus acciones. "Mi primo me llevó a casa inconsciente, en calidad de bulto a las 2:00 de la mañana".

Unas horas después al despertar en su cama, estaba desconcertada por lo sucedido "me sentía rara, me bañaba y olía diferente". La mamá de Regina se preocupó al ver a su hija inconsciente, sin recordar parte de la noche. La primera reacción de la madre fue comprarle la pastilla del día siguiente. Al paso de las horas la situación de Regina no mejoró por lo que sus padres la llevaron al hospital. En la revisión, los médicos detectaron "lesiones por agresión sexual”. En el hospital recomendaron acudir al ministerio público a denunciar, así es que su padre las llevó. Al concluir el proceso legal sus padres la "dejaron completamente sola", estaban muy enojados "porque no llegué a la hora que mi madre me había dicho". Una vez más, el asunto se ocultó y condenó a la mujer. A partir de estas experiencias de abuso por parte de sus primos y la negligencia de sus padres, el quiebre en la familia fue irremediable.

\section{En busca de sentido}

El ser humano, es uno de los animales más indefensos del planeta. Sin la ayuda de otros seres sería imposible sobrevivir, aunque los humanos no sólo necesitan que les provean sus necesidades básicas, también requieren del afecto (Esquivel et al., 2012). Regina encontró ese amor en su abuelo materno. Ella recuerda: "me acompañó por mis papeles cuando estuve en el cuadro de honor". Lamentablemente, su abuelo murió cuando ella tenía 16 años, pero sus consejos y bellos momentos siguen vigentes. Me decía, "sigue adelante, no te quedes". Mi abuelo trabajaba en el periódico. Cuando salió en la portada mi banda favorita no dudo en regalármela, me hacía sentir especial". Regina encontró en su abuelo un sostén que la contuvo 
ante situaciones adversas, al igual que su amigo Escamilla quien le brindó un soporte incondicional en el trabajo. Tanto su abuelo como su amigo le brindaron los factores resilientes necesarios, como el apoyo moral que fue negado por sus padres. Con pesar recuerda: "cuando terminé mis estudios de Diseñadora Gráfica obtuve el primer lugar de aprovechamiento. Mi padre no llegó a la ceremonia del homenaje, lo vimos hasta que estábamos afuera del teatro". Ese día, Regina aprendió a no contar con la presencia de toda su familia, se percató que en lugar de algunos miembros de su familia, tenía la compañía de su red de apoyo. "Aquel día sentí muy bonito, me di cuenta que en realidad no necesitaba tener a mi familia. El que otras personas valoraran mis logros, fue satisfactorio". Regina se siente agradecida con la vida, tiene sus ideas firmes y fue capaz de adaptarse y superar la adversidad a través de sus características personales y redes de apoyo. Algún día dejará su familia de origen, comenta: "no me quiero quedar aquí, mi meta es aplicar para una beca en Corea del Sur".

\section{CONCLUSIONES}

Con base en los resultados presentados, coincidimos con que, tanto los sujetos como las familias son estudiados en función de sus pautas de organización y aspectos constitutivos. De acuerdo Gallego, Pino, Álvarez, Vargas y Correa (2019), la importancia de conocer las características, radica en el fenómeno que representa la familia como fuente de salud o enfermedad.

La novela nos permitió adentrarnos en la dinámica familiar y evidenció como las acciones cotidianas sutilmente posicionaron a la heredera del nombre del abuelo paterno como la hija consentida, la que tuvo libertad de transgredir las reglas y aun así, ser cuidada como la joya de la familia. Los progenitores mostraron diferencias significativas entre sus hijas, la 
consentida y la no querida que fue ignorada, descuidada y desprotegida. Tales negligencias pusieron a la protagonista a merced de familiares rapaces, primos y tías sin escrúpulos que crearon vínculos patológicos para abusar. Por lo que concuerda con el estudio realizado Pinheiro y Fortunato (2019), quienes postulan que, dependiendo de la dinámica familiar, este núcleo puede ser un factor de riesgo para la ocurrencia de rechazo y violencia.

Franco y Ramírez (2016) manifiestan que la negligencia familiar es una de las formas de violencia menos evidenciadas y expresadas. En la novela, se hizo evidente la negligencia familiar por el bajo nivel de afecto, la práctica de crianza deficiente, así como, la complicidad y la negligencia de los progenitores ante el abuso. Lo que posicionó a la participante en una situación de vulnerabilidad y riesgo por el rol que ocupa en el núcleo familiar.

Sin embargo, a pesar de su exposición a situaciones de rechazo por parte de sus familiares, logró buscar sustitutos como el abuelo materno y sus amigos. Desarrolló fuertes lazos de amistad ampliando su red de apoyo. Alejándose así del sentimiento de abandono y soledad vividos. Desarrolló resistencia y la capacidad de reconstrucción ante situaciones muy complicadas como el abuso. Lo que concuerda con los estudios realizados por González-Arratia (2016) acerca del proceso dinámico de resistir, que implica que el traumatizado se sobrepone y reconstruye.

Con respecto a la resiliencia, en esta investigación se entiende como la capacidad de recuperarse y mantener una conducta adaptativa luego de una situación de riesgo (Cala, 2020). Lo que coincide con lo presentado en la novela, la protagonista continuó sus estudios, trabajó para lograr su 
cometido, se graduó como Diseñadora Gráfica y obtuvo el primer lugar de aprovechamiento. Se recuperó después del abuso sexual de los primos y la negligencia de los padres, así como de la falta de apoyo económico para continuar sus estudios. La resiliencia se construyó a partir de las fortalezas que desarrolló Regina como la perseverancia ante la adversidad, el trabajo arduo, las relaciones positivas, la búsqueda de una mejor vida y la visión positiva del futuro.

En la concepción que enmarca la psicología positiva de comprender los procesos y mecanismos que subyacen a las fortalezas y virtudes del ser humano ante eventos desafortunados (González-Arratia \& Medina, 2012). Es imprescindible hacer hincapié en la resiliencia de la protagonista ante los abusos familiares de los padres y abusos sexuales por parte de los primos. Desde la psicología positiva la protagonista nos permite ver sus fortalezas y virtudes desarrolladas ante las adversidades de su vida.

Por ello, se precisa profundizar en esta línea de investigación. Es necesario seguir explorando la potencialidad que tiene el constructo de la resiliencia como herramienta para ayudar, a las personas o grupos a resolver problemas de salud y mejorar la calidad de vida. Además, se sugiere el diseño de programas de intervención puntuales focalizados en la psicología positiva que potencien la resiliencia en estos adolescentes que coadyuven a una mejor percepción de su calidad de vida, tanto a corto como a largo plazo, ya que los costos y efectos psico emocionales y sociales del abuso sexual pueden llegar a extenderse a lo largo de toda la vida de la víctima si no se interviene a tiempo y si no se cuenta con suficientes recursos resilientes.

Como comentario final, concluimos que la limitante de este estudio es el diseño de caso único. Lo que aquí se interpreta no puede ser generalizado. 


\section{Los resultados impulsan a continuar con la realización de otras entrevistas} que permitan clarificar el efecto de las adolescentes víctimas de abuso y rechazo familiar. Conocer la organización constitutiva familiar, el tipo de crianza, las vicisitudes de los adolescentes y los factores de protección. Cabe destacar que este artículo presenta el resultado preliminar de la faceta cualitativa de una investigación.

\section{REFERENCIAS}

\ Agudelo M. E. (2010). Dinámica familiar y salud mental de niños y adolescentes en familias que han vivido las separación de los padres. Revista de la Facultad de Trabajo Social UPB, 24(24), 45-64.

I Astorga, C. M., Castro, F. V., Cabaco, A. S., \& Velasco, E. M. (2014). La Psicología Positiva: antigua o nueva concepción. International Journal of Developmental and Educational Psychology, 3(1), 439-450. Doi:10.17060/ijodaep.2014.n1.v3.522

\ Balcázar, P., González-Arratia, L. F. N. I., Gurrola, G. M., \& Moysén, A. (2013). Investigación cualitativa. ( $2^{a}$ ed.). México: Universidad Autónoma del Estado de México.

I Cala, M. L. P. (2020). La salud desde la perspectiva de la resiliencia. Archivos de Medicina Manizales, 20(1), 203-216.

I Cuevas, Y. (2016). Recomendaciones para el estudio de representaciones sociales en investigación educativa. Cultura y representaciones sociales, 11(21), 109-140.

I Domhardt, M., Münzer, A., Fegert, J. M., \& Goldbeck, L. (2015). Resilience in Survivors of Child Sexual Abuse: A Systematic Review of the Literature. Trauma, violence \& abuse, 16(4), 476-493. Doi: $10.1177 / 1524838014557288$

I Esquivel, V., Faur, E., \& Jelin, E. (2012). Hacia la conceptualización del cuidado: familia, mercado y estado. En V. Esquivel, E. Faur., \& E. Jelin (Eds.), Las lógicas del cuidado infantil. Entre las familias, el estado y el mercado (pp.11-43). Ides.

$\checkmark$ Fondo de las Naciones Unidas para la Infancia. (2019). Panorama Estadístico de la Violencia contra Niñas, Niños y Adolescentes. Recuperado de https://www.unicef.org/mexico/media/1731/file/UNICEF\%20 PanoramaEstadistico.pdf

I Franco, A., \& Ramírez, L. (2016). Abuso sexual infantil: perspectiva clínica y dilemas ético-legales. Revista colombiana de psiquiatría, 45(1), 51-58. Doi:10.1016/j.rcp.2015.07.003

\Gallego , A. M., Pino , J. W., Álvarez , M. M., Vargas, E. D., Correa, L. V. (2019). La dinámica familiar y estilos de crianza: pilares fundamentales en la dimensión socioafectiva. Hallazgos, 16(32), 131-150. Doi: $10.15332 / 2422409 x .5093$

\García, M., Rivera, S., \& Reyes, I. (2014). La percepción de los padres sobre la crianza de los hijos. Acta Colombiana de Psicología, 17, (2), 133-141. 
I González - Arratia, L., N. I. (2016). Resiliencia y personalidad en niños y adolescentes. México: Universidad Autónoma del Estado de México.

I González-Arratia, L.N.I., \& Valdés, J. L. . (2012). Optimismo-pesimismo y resiliencia en adolescentes de una universidad pública. Revista Científica Multidisciplinaria de Prospectiva, 19(3), 207-214. Recuperado de https://www.redalyc.org/pdf/104/10423895002.pdf

$\checkmark$ Instituto Nacional de Estadística y Geografía. (2019). Encuesta Nacional de Victimización y Percepción sobre Seguridad Pública. Recuperado de https://cutt.ly/ZhUFNny

I Losada, A. V. (2015). Familia y psicología. Buenos Aires:Editorial Dunken.

I Noriega, G., Arjona, B. A., \& Noriega, G. A. (2015). La resiliencia en la educación, la escuela y la vida. Perspectivas docentes, (58), 42-48. https://dialnet.unirioja.es/servlet/articulo?codigo=6349252

I Organización Mundial de la Salud.(2020, 8 de junio).Violencia contra los niños. Recuperado de https:// cutt.ly/DhUF1XA

I Pinheiro,A. \& Fortunato, C. (2019). Family Dynamics of Boys Victims of Sexual Abuse. Paidéia, 29. Doi:10.1590/1982-4327e2908

I Piña, J. A. (2015). Un análisis crítico del concepto de resiliencia en psicología. Anales de Psicología, 31(3), 751-758. Doi:10.6018/analesps.31.3.185631

I Quiceno, J. M., Mateus, J., Cardenas, M., Villareal, D., \& Vinaccia, S. (2013). Calidad de vida, resiliencia e ideación suicida en adolescentes víctimas de abuso sexual. Revista de Psicopatología y Psicología Clínica, 18(2), 107-117. Doi:10.5944/rppc.vol.18.num.2.2013.12767

I Remedi, E. (2004). Instituciones educativas. Sujetos, historia e identidades. México: Plaza y Valdés.

ISánchez, G., Aguirre, M., Solano, N., \& Viveros, E. (2015). Sobre la dinámica familiar. Revisión documental. Cultura Educación y Sociedad 6(2), 117-138. Recuperado de http://hdl.handle.net/11323/3107

I Schützenberger, A. (2006). ;Ay, mis ancestros! Omeba.

I Timmerman, M. C., \& Schreuder, P. R. (2014). Sexual abuse of children and youth in residential care: An international review. Aggression and violent behavior, 19(6), 715-720. Doi:10.1016/j.avb.2014.09.001

I Villegas, M., \& González, D. (2011). La investigación cualitativa de la vida cotidiana: medio para la construcción de conocimiento sobre lo social a partir de lo individual. Psicoperspectivas, 10(2), 35-59. Doi:org/fm $3 \mathrm{w}$

I Villegas, M., \& Mallor, P. (2017). Heidi. Un caso de resiliencia al abuso sexual. Revista de Psicoterapia, $106(28), 87-111$.

Envío a dictamen: 4 de febrero de 2021

Reenvío: 19 de abril de 2021

Aprobación: 14 de mayo de 2021 
Brissia Stephanie Fuentes Carmen. Licenciada en Psicología con mención honorífica por la Universidad Autónoma del Estado de México, CU Zumpango. Es estudiante de la Maestría en Psicología de la Salud Orientada a la Investigación en el Instituto de Ciencias de la Salud que se encuentra en el Padrón del Programa Nacional de Posgrados de Calidad de CONACYT en la Universidad Autónoma del Estado de Hidalgo. Correo electrónico: briss0808@gmail.com

Santos Noé Herrera Mijangos. Licenciado en Psicología Social por la Universidad Autónoma Metropolitana, Maestro en Psicología por la Universidad del Valle de México, Maestro en Terapia Gestalt por el Instituto de Terapia Gestalt y Doctor en Ciencias por el Departamento de Investigaciones educativas del CINVESTAV. Con respecto a sus publicaciones, el Dr. Herrera ha publicado 20 artículos científicos, 17 capítulos de libros y 8 memorias en áreas de la Psicología clínica, social y educativa, asimismo a lo largo de su carrera profesional, el Dr. Herrera ha obtenido dos premios nacionales como Director y Codirector de tesis. Actualmente, se encuentra en la Universidad Autónoma del Estado de Hidalgo, en el área de Psicología del Instituto de Ciencias de la Salud como Profesor Investigador de Tiempo Completo. Correo electrónico: psicologonoe@yahoo.com.mx

Andromeda Ivette Valencia Ortiz. Doctora en Psicología de la Salud por la Universidad Nacional Autónoma de México. Investiga en el campo de las enfermedades crónico degenerativas en la infancia en el área de la psicooncología, obesidad infantil, diabetes, epilepsia, ansiedad, depresión y problemas de conducta entre otros. Desarrolló el programa de Intervención para Niños y sus Familias "El Juego del Optimismo" incorporando principios de la Terapia Cognitivo-Conductual y de Psicología Positiva. Ha dirigido tesis de doctorado, maestría, licenciatura y especialidad en neuropsicología. Es autora de varios libros y capítulos en libros de Psicología de la Salud, ha escrito artículos en revistas científicas y de divulgación. Ha sido encargada de la estandarización a nivel nacional de distintas pruebas psicométricas. Ha organizado y participado en distintos Coloquios y Congresos Nacionales e internacionales; ha trabajado en programas de televisión, radio, prensa escrita y medios electrónicos, de igual forma su participación como conferencista 
es destacada. Actualmente, es Profesora Investigadora de Tiempo Completo "B" (perfil PROMEP) en el Instituto de Ciencias de la Salud (ICSa) de la Universidad Autónoma del Estado de Hidalgo, UAEH y miembro del Cuerpo Académico "Salud Emocional" y Candidata del Sistema Nacional de Investigadores (SNI). Correo electrónico: andromeda valencia@uaeh.edu.mx

Ana Olivia Ruíz Martínez. Miembro del Sistema Nacional de Investigadores Nivel 1. Doctora en Psicología con orientación en psicología y salud, así como, Licenciada en Psicología por la UNAM. Profesora de Tiempo Completo de la Universidad Autónoma del Estado de México, Docente con Perfil deseable PRODEP (SEP) e integrante del cuerpo académico: Cultura y Personalidad de la Facultad de Ciencias de la Conducta de la UAEM, licenciatura y doctorado. Realiza investigación de competencia internacional mediante su línea de investigación: Salud psicológica y factores asociados; abordando temas como: Trastornos alimentarios, obesidad, salud mental, enfermedades crónicas y su relación con factores personales, familiares y socio-culturales. Correo electrónico: ana_olivi@yahoo.com.mx 